\begin{tabular}{|c|c|}
\hline Title & A new analysis method for accurate supercurrent distribution inside high-temperature superconducting bulk \\
\hline Author(s) & Y anagawa, Tomohiro; Kaneda, Kazufumi; Ishiyama, A tsushi; Noguchi, So; Y amashita, Hideo \\
\hline Citation & $\begin{array}{l}\text { IEEE transactions on magnetics, 42(4), 751-754 } \\
\text { https:/doi.org/10.1109// MA G.2006.871606 }\end{array}$ \\
\hline Issue Date & $2006-04$ \\
\hline Doc URL & http://hdl.handle.net/2115/8522 \\
\hline Rights & $\begin{array}{l}\text { (c) } 2006 \text { IEEE. Personal use of this material is permitted. However, permission to reprint/republish this material for } \\
\text { advertising or promotional purposes or for creating new collective works for resale or redistribution to servers or lists, } \\
\text { or to reuse any copyrighted component of this work in other works must be obtained from the }\end{array}$ \\
\hline Type & article \\
\hline File Information & 01608315.pdf \\
\hline
\end{tabular}

Instructions for use 


\title{
A New Analysis Method for Accurate Supercurrent Distribution Inside High-Temperature Superconducting Bulk
}

\author{
Tomohiro Yanagawa ${ }^{1}$, Kazufumi Kaneda ${ }^{1}$, Atsushi Ishiyama ${ }^{2}$, So Noguchi ${ }^{3}$, and Hideo Yamashita ${ }^{4}$ \\ ${ }^{1}$ Graduate School of Engineering, Hiroshima University, Hiroshima 739-8527, Japan \\ ${ }^{2}$ Department of Electrical, Electronics and Computer Engineering, Waseda University, Shinjuku-ku 169-6555, Japan \\ ${ }^{3}$ Graduate School of Information Science and Technique, Hokkaido University, Sapporo 060-0814, Japan \\ ${ }^{4}$ Graduate School of Engineering, Hiroshima Institute of Technology, Hiroshima 731-5193, Japan
}

It is difficult to accurately simulate supercurrent in high-temperature superconducting bulk even by adopting the finite-element method. The equivalent electrical conductivity of high-temperature superconducting bulk, which has a strong nonlinearity according to the $E-J$ power law, is introduced for the supercurrent analysis. However, the strong nonlinearity results in bad convergency of the nonlinear equation solvers, i.e., the Newton-Raphson (NR) method. Consequently, the unsuitable and/or undulating supercurrent is observed. Therefore, in this paper, the accuracy of the supercurrent analysis in the high-temperature superconducting bulk is discussed and a new method is proposed for the accurate high-temperature superconducting bulk simulation. Then, the method combined a line search with the NR method is adopted as a nonlinear equation solver, and the improvement of convergency and computation time are investigated.

Index Terms-E-J power law, high-temperature superconducting (HTS) bulk, nonlinear equation solver, supercurrent distribution.

\section{INTRODUCTION}

I $\mathrm{N}$ applications of high-temperature superconducting (HTS) bulk, such as flywheels, magnetic levitations, bulk magnets, and so on, it is very important to comprehend the accurate characteristics of HTS bulk. Therefore, it is necessary to estimate the highly accurate Lorentz force, which is calculated from the supercurrent distribution, for design of HTS bulk applications [1], [2]. Consequently, the supercurrent distribution, depending on an external magnetic field and its history, is one of the important HTS bulk characteristics. To simulate the supercurrent, it is treated as an eddy current in normal conductor with a nonlinear equivalent electrical conductivity [1], [3], [4]. By using of the finite-element method (FEM), even with very fine mesh, it occurs that the evaluated supercurrent partly flows in the opposite direction to the theoretical one (see Fig. 1). The simulation results are caused by the strong nonlinearity of the equivalent electrical conductivity, yielded from the $E-J$ characteristics, as shown in Fig. 2. Therefore, a development or a modification of the highly accurate simulation method for HTS bulk is strongly desired. So, we propose a newly developed numerical simulation method to comprehend the electromagnetic characteristics of HTS bulk and investigate the accuracy compared with the FEM. Moreover, for verification to solve the strong nonlinear problem, the Newton-Raphson (NR) method and a line search (LS) combined with the NR method [5] are compared.

Many methods to simulate the HTS bulk were proposed, i.e., flux flow creep method [6], and Ginzburg-Landau method [7]. However, the microscopic physical parameters in the methods are required. Therefore, we focus on the large-scale simulation

Digital Object Identifier 10.1109/TMAG.2006.871606

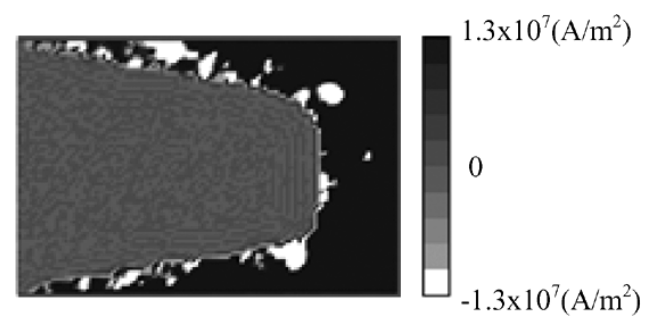

Fig. 1. Supercurrent distribution by the common FEM employing the $E-J$ power law $(t=2.0 \mathrm{~s})$.

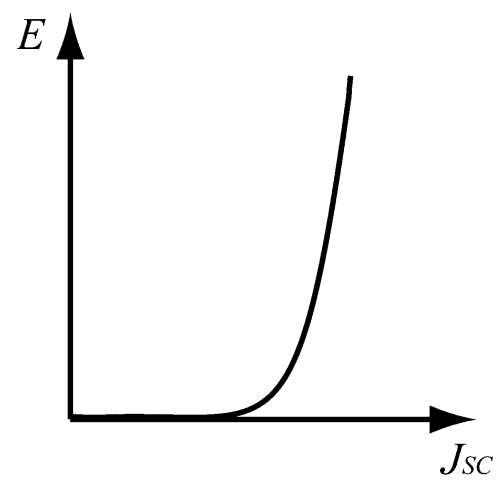

Fig. 2. $E-J$ characteristics of high-temperature superconductor.

methods, such as FEM, which needs the macroscopic physical characteristics, i.e., $n$ value, since they are easily measurable.

\section{Mathematical Model}

To simulate a space including the HTS bulk, the governing equation derived from the Maxwell's equations is

$$
\operatorname{rot}(\nu \boldsymbol{B})=\boldsymbol{J}_{0}+\boldsymbol{J}_{\mathrm{SC}}
$$


where $B, \nu$, and $\boldsymbol{J}_{0}$ are the magnetic flux density, the reluctivity, and the source current density, respectively. The supercurrent density $\boldsymbol{J}_{\mathrm{SC}}$ is calculated by the following relations:

$$
\begin{aligned}
& \boldsymbol{J}_{\mathrm{SC}}=\sigma_{\mathrm{SC}} \boldsymbol{E} \quad \text { and } \\
& \boldsymbol{E}=\frac{\partial \boldsymbol{A}}{\partial t}
\end{aligned}
$$

where $\sigma_{\mathrm{SC}}, \boldsymbol{E}, \boldsymbol{A}$, and $t$ are the equivalent electrical conductivity, the electrical fields, the magnetic vector potential, and the time, respectively. Moreover, the accurate supercurrent density simulation in HTS bulk is achieved by taking account of the $E-J$ power law

$$
\boldsymbol{E}=E_{\mathrm{C}}\left(\frac{\left|\boldsymbol{J}_{\mathrm{SC}}\right|}{J_{\mathrm{C}}}\right)^{n}
$$

where $J_{\mathrm{C}}$ and $E_{\mathrm{C}}$ are the critical current density $\left(=1.25 \times 10^{7}\right.$ $\left.\mathrm{A} / \mathrm{m}^{2}\right)$ and the electric field criterion $\left(=10^{-7} \mathrm{~V} / \mathrm{mm}\right)$ that defines $J_{\mathrm{C}}$, respectively, and $n$ ( $=20$, in this paper) is the index number, called " $n$ value," which concerns the strength of the nonlinearity. Here, the equivalent electrical conductivity is derived from

$$
\sigma_{\mathrm{SC}}=\frac{J_{\mathrm{C}}}{\boldsymbol{E}}\left(\frac{\boldsymbol{E}}{E_{\mathrm{C}}}\right)^{1 / n}
$$

These equations are to be solved numerically as the nonlinear problem.

\section{PROPOSED METHOD}

By using the weight function $W$ (1) yields

$$
\iint_{\Omega} W\left\{\operatorname{rot}(\nu \boldsymbol{B})-\boldsymbol{J}_{0}-\boldsymbol{J}_{\mathrm{SC}}\right\} \mathrm{d} \Omega=0 .
$$

In the FEM employing the linear triangular element, the magnetic flux density $\boldsymbol{B}$ in (6) is substituted for

$$
\boldsymbol{B}_{\text {element }}^{(e)}=\operatorname{rot}\left(\sum_{i=1}^{3} w_{i} A_{i}\right)
$$

where $B_{\text {element }}^{(e)}$ represents the magnetic flux density in the element $e$, and $w_{i}$ and $A_{i}$ are the weight function and the vector potential at node $i$. Then, the equations obtained from (6) and (7) are solved.

In the proposed method, the magnetic flux density $\boldsymbol{B}$ in (6) is substituted for the magnetic flux density $\boldsymbol{B}_{\text {propose }}$ obtained as to each node from their neighboring elements. That is, $B_{\text {propose }}$ is obtained by

$$
\boldsymbol{B}_{\text {propose }}=\sum_{i=1}^{3} w_{i} \boldsymbol{B}_{\text {node }}^{(i)} \text {. }
$$

Here, $\boldsymbol{B}_{\text {node }}^{(i)}$ is yielded from

$$
\boldsymbol{B}_{\text {node }}^{(i)}=\frac{\sum_{e=1}^{m} B_{\text {element }}^{(e)}}{m}
$$

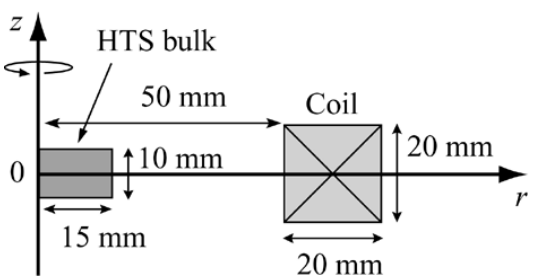

Fig. 3. Analytical model consisting of an HTS bulk and an exciting coil.

where the magnetic flux density on elements, $\boldsymbol{B}_{\text {element }}^{(e)}$, which is also calculated from (7), and $m$ is the number of the neighboring elements which connect with node $i$. In the proposed method, the equations obtained from substituting $B$ in (6) for $B_{\text {propose }}$ in (8) are to be solved as the vector potential $\boldsymbol{A}$ is unknown.

The difference between the FEM and the proposed method is in the way of the evaluation of the $\boldsymbol{B}$ field. The $\boldsymbol{B}$ obtained from (7) is constant on each element in the FEM with linear triangle element, while $\boldsymbol{B}$ in (8) linearly varies in each element. The constant value of $\boldsymbol{B}$ is considered as one of the factors to lead to the unsuitable result. In the proposed method, therefore, the nodal values of $B$, which are evaluated from their neighboring elements, are introduced, and the $B$ in each element is approximated as a liner function by the different way from the quadratic triangle element. In consequence, the magnetic flux density $B$ is evaluated accurately. In addition, to obtain the accurate magnetic flux density conducts the accurate magnetic vector potential $\boldsymbol{A}$ and the supercurrent density $\boldsymbol{J}_{\mathrm{SC}}$.

This formulation enables to obtain the suitably smooth magnetic vector potential $\boldsymbol{A}$ with high accuracy in regard to the strong nonlinear $\sigma_{\mathrm{SC}}$.

\section{APPLICATION AND RESUlTS}

In order to confirm the validity and the utility of the proposed method, an HTS bulk simulation was carried out. The axially symmetrical analysis model consists of an HTS bulk disk and an exciting coil as shown in Fig. 3. In the proposed method, the nodal linear triangular element is employed, and all the simulation is performed with the same mesh. The numbers of nodes and elements are 11634 and 23072 , respectively. In addition, the number of elements of the HTS bulk is 13068 .

\section{A. Bean Model}

At first step, the critical state model (bean model), which corresponds to $n=\infty$ in (5), was employed in the simulation. Here, the way to simulate the bean model is proposed by [8]. The coil current monotonously and slowly increases from 0 at $80 \mathrm{~A} / \mathrm{s}$, and the analytical time interval of $\Delta t$ is 1.0 . In the FEM, the nodal linear and quadratic triangle elements are tested for comparing the proposed method. The convergence properties of the NR in both the proposed method and the FEM are not bad for the bean model. The supercurrent density inside of the HTS bulk on $z=0$ at $t=10 \mathrm{~s}$, obtained by the proposed method and 


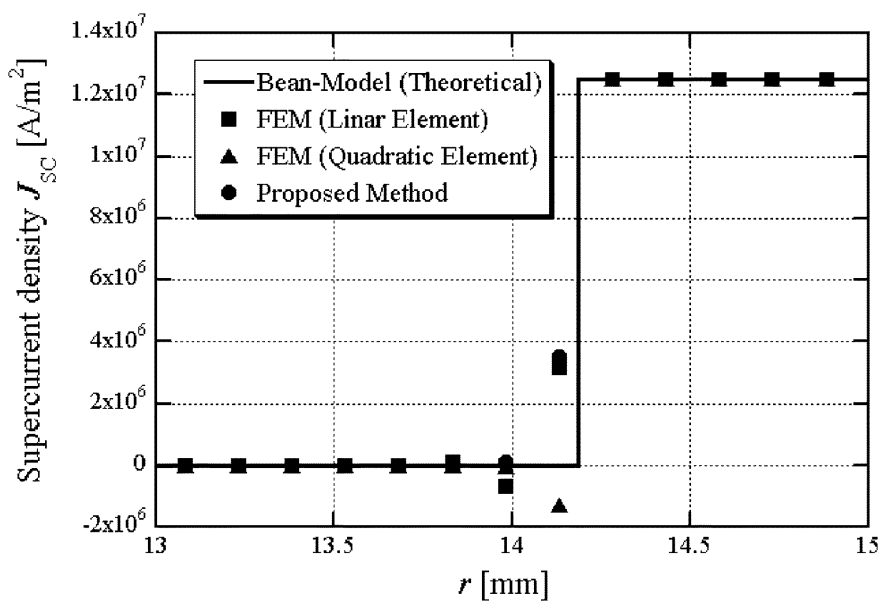

Fig. 4. Supercurrent density inside HTS bulk employing the Bean model $(z=$ $0, t=10 \mathrm{~s})$.

the FEM, are shown in Fig. 4. The unsuitably undulating supercurrent densities of both the linear and the quadratic FEMs are observed in the range of $r=13.8-14.2 \mathrm{~mm}$ on Fig. 4 . On the other hand, the supercurrent density of the proposed method suitably smoothly varies. Moreover, a good agreement between the theoretically obtained supercurrent density [8] and that obtained from the proposed method is also revealed from Fig. 4.

\section{B. E-J Power Law Model}

At second step, in order to take account of the time-varying external magnetic field, the $E-J$ power law (4) was employed in the simulation. The coil current monotonously and quickly increases from 0 at $2240 \mathrm{~A} / \mathrm{s}$, and the analytical time interval $\Delta t$ is $0.05 \mathrm{~s}$. To solve the nonlinear problem, the NR method and the method in [5] (which combines a LS with the NR method) are adopted, and the convergence characteristics are investigated.

The convergence criterion of the nonlinear equation solver is adopted as follows:

$$
\left|\frac{\delta \boldsymbol{A}^{(k)}}{\boldsymbol{A}^{(k+1)}}\right|<1.0 \times 10^{-3}
$$

where $k$ is the number of the nonlinear iteration. Note, in the case that the magnetic vector potential $A$ is nearly 0 , the convergence criterion is adopted as follows:

$$
\left|\delta A^{(k)}\right|<1.0 \times 10^{-25}
$$

The result of the FEM is shown in Fig. 1; the reasonable supercurrent distribution could not be obtained, obviously.

The supercurrent distributions inside of the HTS bulk at $t=$ $0.05,0.5,1.0,1.5,2.0,2.5 \mathrm{~s}$, obtained by the proposed method with the LS method, are shown in Fig. 5. There are no differences between the results of the proposed method with both the LS and the NR methods. The unsuitably undulating supercurrent density in the distributions in Fig. 5 is not observed at all.

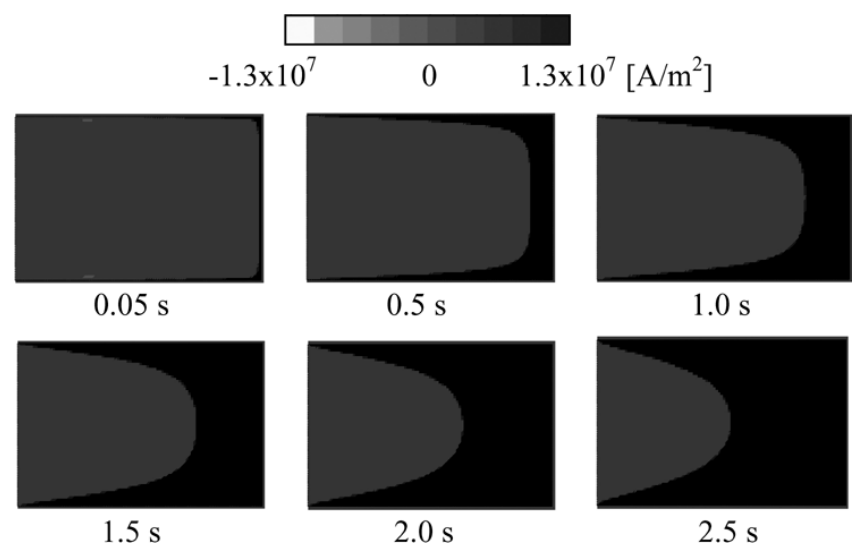

Fig. 5. Supercurrent distribution obtained by the proposed method with the LS method ( $E-J$ power law model).

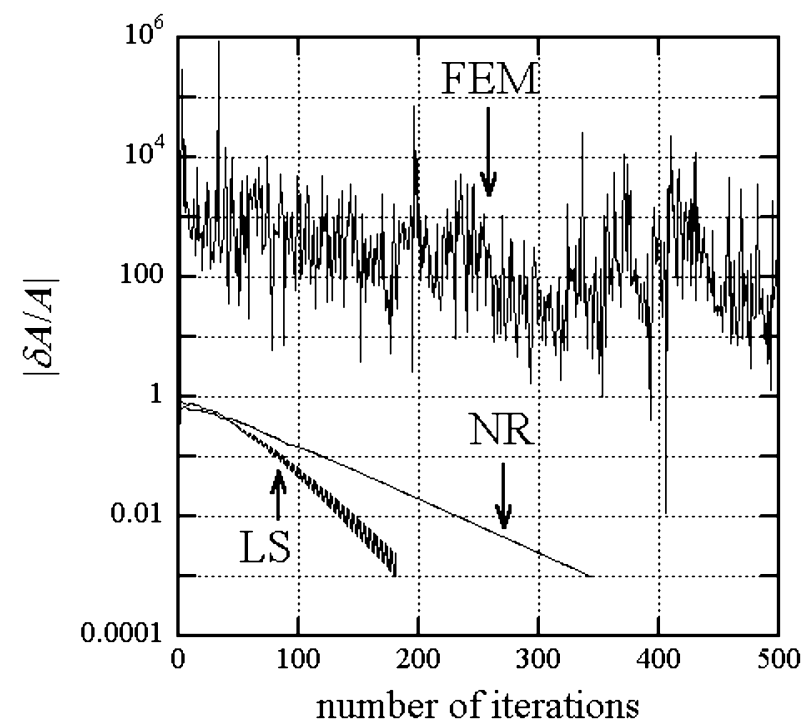

Fig. 6. Convergence characteristics of the nonlinear iterative $(t=0.05 \mathrm{~s})$. The two lines of LS and NR are obtained by the proposed method, and the FEM employing the LS method are plotted ( $E-J$ power law model).

\section{DISCUSSION}

\section{A. Proposed Method and FEM}

The validity of the proposed method is confirmed by comparison of the supercurrent density with the theoretically obtained one, as shown in Fig. 4.

On the other hand, the convergence property of the NR in the FEM is obviously bad for the $E-J$ power law model, as shown in Fig. 6. In the FEM, the convergence criterion was never satisfied without reference to employing the LS method or the common NR method. Consequently, the magnetic vector potential $\boldsymbol{A}$ and the supercurrent density $\boldsymbol{J}_{\mathrm{SC}}$ cannot be evaluated. Therefore, in this attempt, the iterative computation of the nonlinear solver only in the FEM is stopped at 500 times, even if the convergence does not reach in order to proceed the time step. In this simulation model, the supercurrent distribution has to be symmetric against the $r$ axis. However, the supercurrent distribution obtained from the FEM with unsuitable 


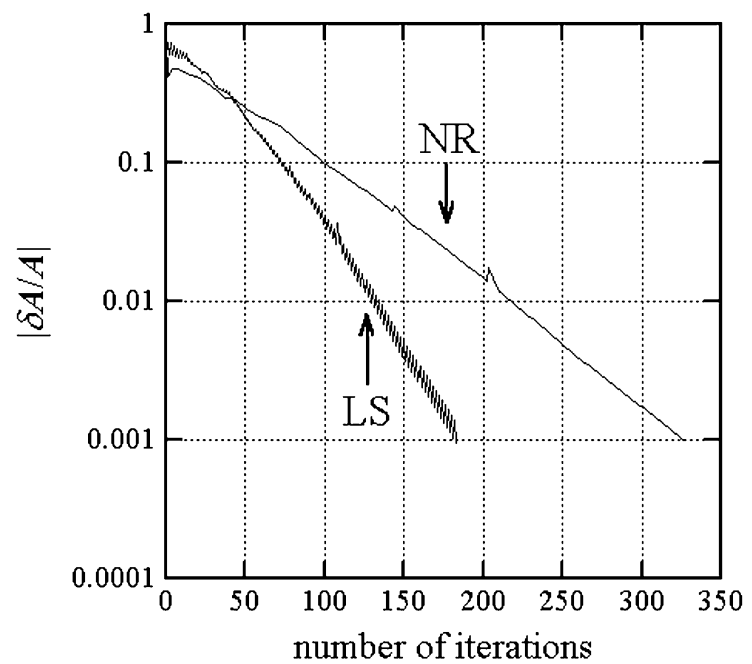

Fig. 7. Convergence characteristics of the nonlinear iterative $(t=2.50 \mathrm{~s})$. The two lines of NR and LS are obtained by proposed method ( $E-J$ power law model).

TABLE I

Computation Time of Nonlinear Solvers ( $E-J$ Power LaW Model)

\begin{tabular}{c|c|c|c}
\hline \hline Solver & $\begin{array}{c}\text { Total computation } \\
\text { time [min] } \\
\text { (normalized) }\end{array}$ & $\begin{array}{c}\text { Average of the } \\
\text { computation time } \\
\text { per time step } \\
\text { (normalized) }\end{array}$ & $\begin{array}{c}\text { Average of the } \\
\text { number of } \\
\text { nonlinear itera- } \\
\text { tions per time step } \\
\text { (normalized) }\end{array}$ \\
\hline NR & $4971(1.00)$ & $99.4(1.00)$ & $351(1.00)$ \\
\hline LS & $2927(0.59)$ & $58.5(0.59)$ & $195(0.56)$ \\
\hline \hline
\end{tabular}

convergence criterion was asymmetrical (see Fig. 1) because of the bad convergence characteristics.

In the proposed method, the applied convergence criterion was satisfied on each time step in the both case employing the LS and the NR, as shown in Figs. 5 and 7, and the suitably smooth supercurrent distributions were obtained. It is considered that the results shown in Fig. 5 are fully reasonable, since the bean model result of the proposed method agrees with the theoretical one (see Fig. 4). In order to investigate in detail, the investigation by the experiments may be carried out.

\section{B. LS and NR Method}

Two kinds of nonlinear equation solvers for the FEM and the proposed method are adopted, and the improvement of the convergence characteristics is investigated. As shown in Fig. 6, the convergence characteristic of the FEM was not improved even by employing the LS method.

On the other hand, the number of iterations in the proposed method employing the LS method is smaller than that of the common NR method (see Figs. 5 and 7). Table I shows the computation time and the number of iterations of the proposed method employing each nonlinear equation solver. The "total computation time" means the time required to the simulation from $t=0.05$ to $2.5 \mathrm{~s}$. The "average of the computation time per time step" means an average time of "total computation time" per time step. The "average of the number of nonlinear iterations" means the average number of the nonlinear iterations per time step. As shown in Table I, the computation time of the proposed method is reduced to $60 \%$ by employing the LS method.

From the results, the effect of the LS method is confirmed to the proposed method. However, the convergence characteristic of the FEM cannot be improved. Consequently, the utility of the proposed method was confirmed from the point of view of the effect of the LS method.

\section{CONCLUSION}

In this paper, we have proposed the method for highly accurate HTS bulk simulation and confirmed its validity as compared with the FEM and the theoretical analysis. The suitable result in the case of the critical state model (bean model) was obtained, and it agreed with the theoretical solution well.

For the $E-J$ power law model, the proposed method was applied. The supercurrent distributions obtained by the proposed method are suitably smooth, while that obtained by the FEM are unsuitable and undulate because of the bad convergence. The convergence characteristic of the proposed method was considerably improved as compared with the FEM. Consequently, the convergence criterion (that cannot be done in the FEM) can be configured and the suitable supercurrent distribution can be obtained.

Finally, the improvement of the convergence characteristic has been investigated by adopting the LS method. The improvement was confirmed to the proposed method, while there is no effect of the LS method to the FEM.

\section{REFERENCES}

[1] J. Natatsugawa, S. Noguchi, A. Ishiyama, H. Kado, and M. Ichikawa, "Magnetic characteristics of high-Tc superconducting fault current limiter," IEEE Trans. Appl. Supercond., vol. 9, no. 2, pp. 1373-1376, Jun. 1999.

[2] M. Tsuda, K. Tsuchiya, N. Harada, and T. Hamajima, "Dependence of lateral stiffness on magnetic field distribution at field-cooling process of HTS bulk system," IEEE Trans. Appl. Supercond., vol. 14, no. 2, pp. 948-951, Jun. 2004.

[3] M. Tsuda, H. Lee, S. Noguchi, and Y. Iwasa, "“electromaglev" ("activemaglev"- magnetic levitation of a superconducting disk with a DC field generated by electromagnets. Par 4: Theoretical and experimental results on supercurrent distributions in field - cooled YBCO disks," Cryogenics, vol. 39, pp. 893-903, 1999.

[4] H. Shimizu, H. Ueda, M. Tsuda, and A. Ishiyama, "Trapped field characteristics of $\mathrm{Y}-\mathrm{Ba}-\mathrm{Cu}-\mathrm{O}$ bulk in time-varying external magnetic field," IEEE Trans. Appl. Supercond., vol. 12, no. 1, pp. 820-823, Mar. 2002.

[5] K. Fujiwara, Y. Okamoto, A. Kameari, and A. Ahagon, "The Newton-Raphson method accelerated by using a line search-comparison between energy functional and residual minimization," presented at the IEE Jpn. Tech. Meeting Magnetics, 2004.

[6] A. Kamitani, S. Ohshima, and T. Yokono, "Magnetic shielding analysis of axis symmetric HTS plate by flux flow creep model," IEEE Trans. Appl. Supercond., vol. 9, no. 2, pp. 3050-3053, Jun. 1999.

[7] K. Demachi, Y. Yoshida, H. Asakura, and K. Miya, "Numerical analysis of magnetization processes in type II superconductors based on Ginzburg-Landau theory," IEEE Trans. Magn., vol. 32, no. 3, pp. 1156-1159, May 1996.

[8] H. Hashizume, T. Sugiura, K. Miya, and S. Toda, "Numerical analysis of electromagnetic phenomena in superconductors," IEEE Trans. Appl. Supercond., vol. 28, no. 2, pp. 1332-1335, Jun. 1992.

Manuscript received June 25, 2005 (e-mail: noguchi@ssi.ist.hokudai.ac.jp). 